\title{
Inferior ischemic ventricular septal defect: Atypical triple pericardial patch implantation technique
}

We presented the successful management of an inferior ischemic ventricular septal defect (VSD) associated with posterior left ventricular free wall rupture, following the occlusion of a dominant right coronary artery (RCA). The defect was approached through a longitudinal incision over the infarcted area, $1 \mathrm{~cm}$ parallel to the posterior descending coronary artery (PDA). The VSD was repaired using three pericardial patches and GRF (gelatin-resorcinol-formaldehyde) glue. ECMO and IABP were used in order to decrease the LV wall tension, the risk of recurrence of VSD and the risk of free wall rupture. The postoperative echocardiogram documented good biventricular geometry with a left ventricle ejection fraction (LVEF) of 55\%. No left-to-right shunt was seen.

Keywords: Ischemic ventricular septal defect - Patch repair - Myocardial infarction - Free wall rupture
Federico Ranocchi, Marco Picichè, Mazia Cottini*, Emilio Ferretti and Francesco Musumeci

Department of Heart and Vessels, Cardiac Surgery Unit and Heart Transplantation Center, S CamilloForlanini Hospital, 00152 Rome, Italy *Author for correspondence:

Tel: +390658704857

E-mail: marzia.cottini@gmail.com Submitted: September 27, 2017 Accepted: October 23, 2017

Published online: October 28, 2017

\section{Introduction}

An ischemic ventricular septal defect (iVSD) is a rare but life-threatening complication of myocardial infarction. The incidence in the pre-thrombolytic era was approximately 1 to 3 percent, but declined following the use of antifibrinolytic agents to 0.2 percent. While in early reports the time interval between MI and the onset of iVSD was about 3 to 5 days, it decreased to around 24 hours after the advent of aggressive reperfusion strategies [1,2]. Risk factors of iVSD are advanced age, female sex, previous smoking, anterior MI, low Killip class and increased heart rate on admission [3]. Despite the improvement of surgical techniques, the mortality-rate is still very high. Proper timing of surgery, an adequate surgical technique and the use of temporary mechanical supports are crucial to reduce mortality in these high risk patients.

\section{Case Report}

A 63-year-old male with a medical history of dyslipidemia and hypertension, was referred to a tertiary hospital due to dyspnoea and chest pain. The electrocardiogram showed sinus tachycardia heart rate of 120-130 bpm, first degree-atrioventricular block and signs of inferior myocardial infarction. The first transthoracic echocardiogram (TTE) just showed a dilated left ventricle (The Laboratory tests revealed elevated troponin-I (6.29 ng/ $\mathrm{ml}$ ), CPK (696 IU/L), N-terminal pro Brain Natriuretic Peptide (NT-proBNP, 1207.4 pg/ $\mathrm{ml})$, and leukocytosis $\left(21.4 \times 10^{3} / \mu \mathrm{L}\right)$. Due to signs and symptoms of congestive heart failure, low oxygen saturation, pulmonary oedema, low arterial blood pressure (ABP), the patient was transferred to the Intensive Cardiology Unit. Coronary angiography disclosed the occlusion of a dominant right coronary artery (RCA) and a critical stenosis of the circumflex artery. The RCA was treated by implanting a drug-eluting stent, and the patient recovered in less than 24 hours. Because of progressive hemodynamic 
instability, a TEE was performed documenting a dilated and hypokinetic left ventricle, Left Ventricle Ejection Fraction (LVEF) 40\% and systolic pulmonary hypertension of $75 \mathrm{mmHg}$, dilatation and hypokinsia of the right ventricle, massive shunting at the inferiormedial-basal side of the interventricular septum (Qp/Qs>2.5), a fragile-thinned posterior LV wall and moderate blood pericardial effusion (Figure 1). Norepinephrine and epinephrine infusions were administered $(0.1 \mathrm{mcg} / \mathrm{Kg} / \mathrm{min})$. Poor haemodynamic condition required the support of an Intra-Aortic Balloon Pump (IABP) (Figures 2-6).

\section{Discussion}

David reported that "repair of postinfarction VSD by infarct exclusion is a relatively simple operative procedure and seems to have improved the results of surgery in patients with posterior VSD. Similarly, the triple patches technique seemed to be useful in maintaining the LV and RV geometry and volume, avoiding the distortion of the papillary muscles and the onset of mitral regurgitation. According to Musumeci et al. [4], the GRF may be safely inserted as a sealant between the septum and the patch as a preventive

\section{A}

B
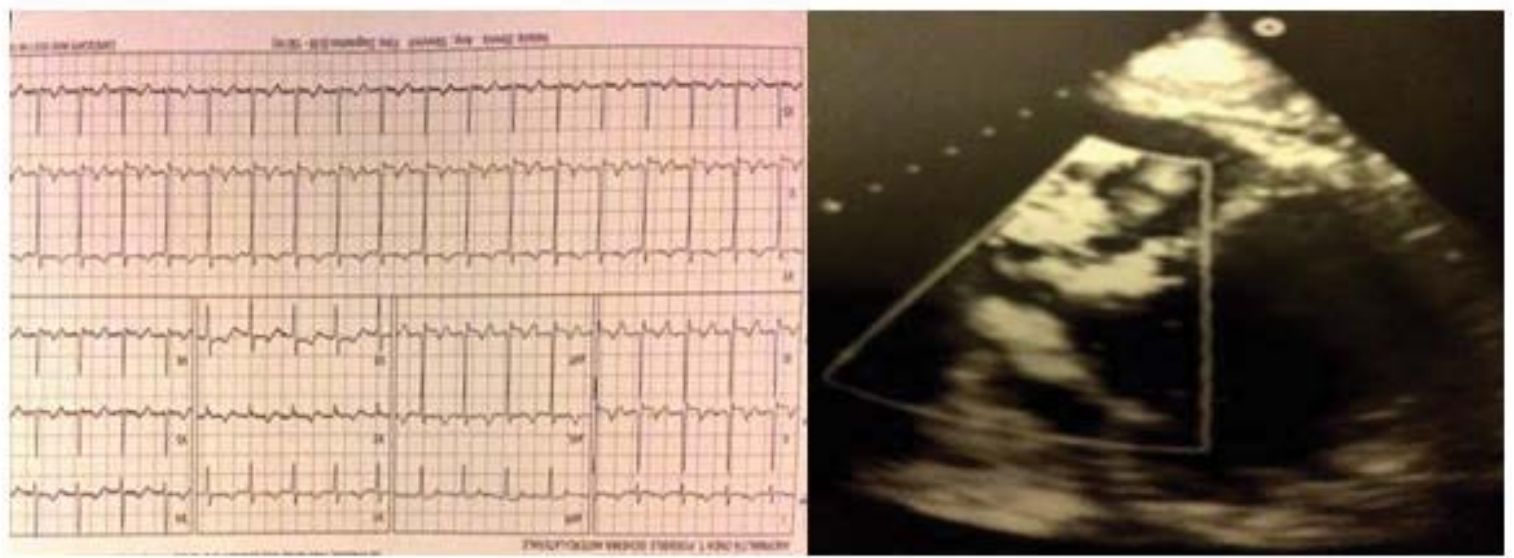

Figure 1: Electrocardiogram showed first degree-atrioventricular block, signs of inferior myocardial infarction (a); transthoracic echocardiogram documented an inferior ventricular septal defect (b).

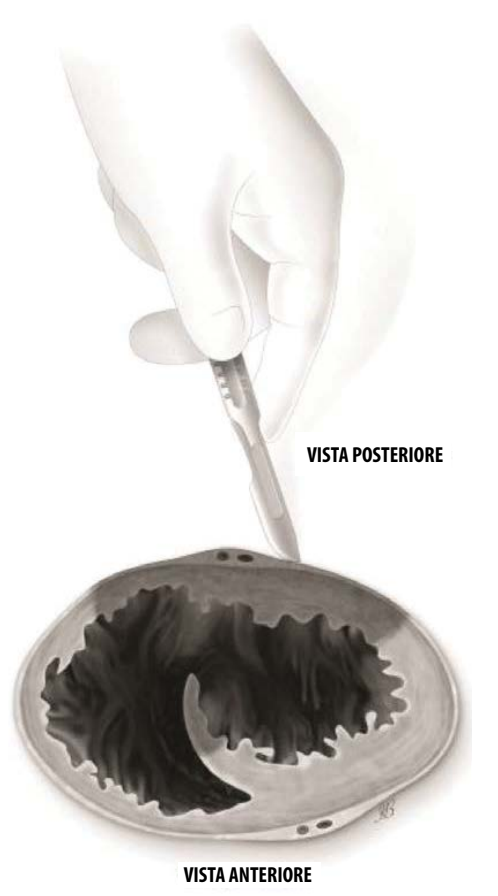

Figure 2: Posterior longitudinal ventriculotomy parallel to the posterior descending artery $(0.5-1 \mathrm{~cm})$, over the infarcted area.

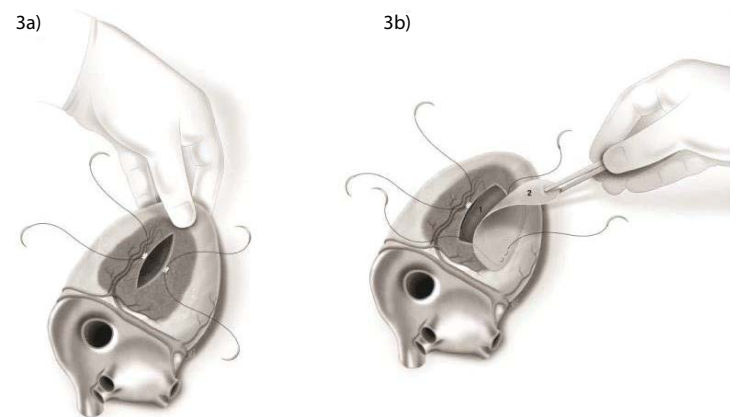

Figure 3: a) Two large oval pericardial patches $(1,2)$ were positioned inside and outside the ventriculotomy covering the LV and RV segments of the infarcted area and of the contiguous myocardium. b) The patches were sutured together with continuous mattress suture (prolene 2-0) next to the basis of the papillary muscles extending towards the apex.

tool against leakage, and between the patches as reinforcement. Moreover, the continued mattress suture seemed to accelerate the procedure compared to the interrupted suture technique. The intraoperative TEE documented hypokhinesia of the right ventricle, and the absence of L-to-R shunt. Veno-arterial 


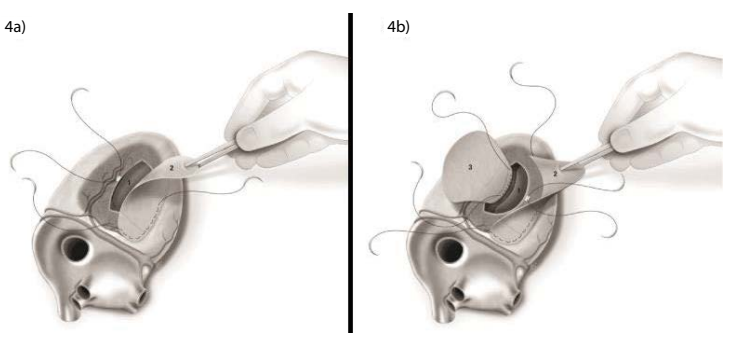

Figure 4: a) A third large oval pericardial patch (3) was sutured from the anterior to the posterior side of the interventricular septum (prolene 2-0); GRF was injected (b). 5a)

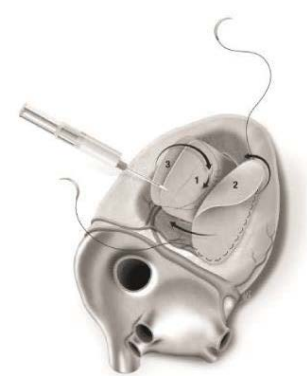

5b)

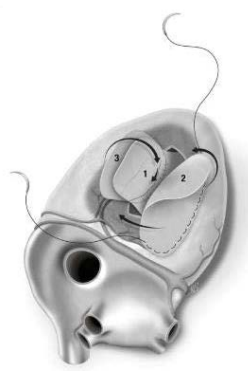

Figure 5: a) The suture used for the anastomosis of patches 1-2 included the septal patch 3, hence excluding the VSD and reinforcing the ischemic postero-lateral side of LV and RV. b) GRF filled the space in between the patches.

extracorporeal membrane oxygenation (VA-ECMO, PLS Set, Bioline Coating, MAQUET) was mandatory in our opinion. An arterial percutaneous cannula was inserted into the ascending aorta (Medtronic,

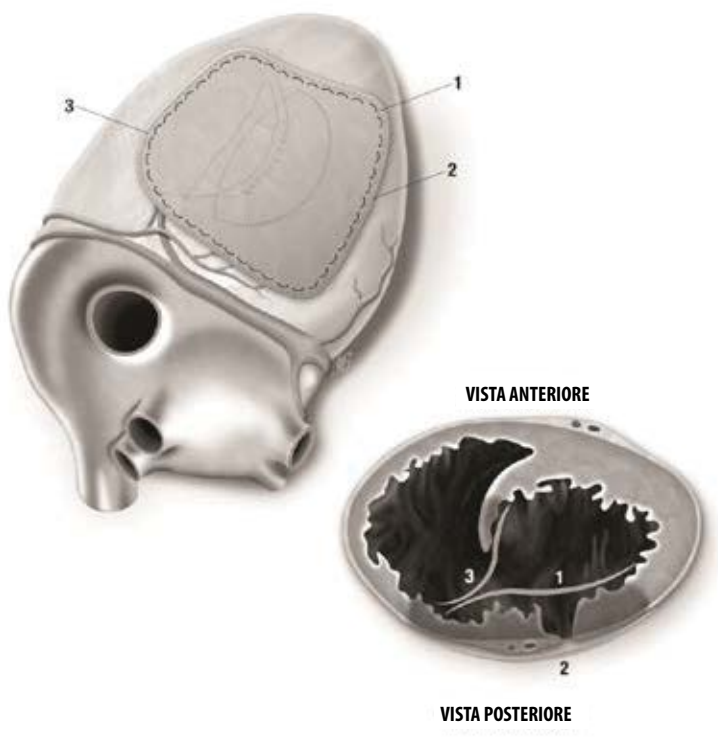

Figure 6: Completed repair.

BioMedicus, $19 \mathrm{Fr}$ ), and a multistage cannula was inserted in the left femoral vein (Medtronic, BIOMEDICUS MS 25 Fr $\times 60 \mathrm{~cm})$. Venting was achieved from the left superior pulmonary vein. We think that VA-ECMO and IABP by reducing the LV volume and the afterload respectively, decreased the tension exerted on to the sutures and the risk of bleeding, and enhanced the possibilities of myocardial recovery. Finally, postoperative echocardiogram showed that the geometry of the ventricles was respected. Our successful case suggests that the three patches technique may be a valuable option with these difficult patients [5-7].

\section{Executive summary}

We presented the successful management of an inferior ischemic ventricular septal defect (VSD) associated with posterior left ventricular free wall rupture, following the occlusion of a dominant right coronary artery (RCA).

The defect was approached through a longitudinal incision over the infarcted area, $1 \mathrm{~cm}$ parallel to the posterior descending coronary artery (PDA). The VSD was repaired using three pericardial patches and GRF (gelatin-resorcinol-formaldehyde) glue. ECMO and IABP were used in order to decrease the LV wall tension, the risk of recurrence of VSD and the risk of free wall rupture.

The postoperative echocardiogram documented good biventricular geometry with a left ventricle ejection fraction (LVEF) of 55\%. No left-to-right shunt was seen. 


\section{References}

1. Gaudiani VA, Miller DC, Stinson EB, et al. Postinfarction ventricular septal defect: an argument for early operation. Surgery. 89: 48-55 (1981).

2. Crenshaw BS, Christopher BG. Risk Factors, Angiographic Patterns, and Outcomes in Patients With Ventricular Septal Defect Complicating Acute Myocardial Infarction. Circulation. 101: 27-32 (2000).

3. Perrotta $S$, Lentini $S$. In patients undergoing surgical repair of post-infarction ventricular septal defect, does concomitant revascularization improve prognosis? Interact. Cardiovasc. Thorac. Surg. 9(5): 879-87 (2009).
4. Musumeci F, Shukla V. Early repair of postinfarction ventricular septal defect with gelatin-resorcin-formol biological glue. Ann. Thorac. Surg. 62(2): 486-488 (1996).

5. Caimmi PP, Grossini E, Kapetanakis EI, et al. Double Patch Repair through a Single Ventriculotomy for Ischemic Ventricular Septal Defects. Ann. Thorac. Surg. 89: 1679-1681 (2010).

6. David TE, Armstrong S. Surgical repair of postinfarction ventricular septal defect by infarct exclusion. Semin. Thorac. Cardiovasc. Surg. 10(2): 105-110 (1998).

7. Okamoto Y, Yamamoto K, Asami F, et al. Early and midterm outcome of triple patch technique for postinfarction ventricular septal defects. J. Thorac. Cardiovasc. Surg. 151: 1711-1716 (2016). 